\title{
Herscovici's Conjecture on the Product of the Thorn Graphs of the Complete Graphs
}

\author{
Dong-Lin Hao $\cdot$ Ze-Tu Gao $\cdot$ Jian-Hua Yin
}

Received: 19 March 2014/Revised: 7 May 2014/Accepted: 7 May 2014/

Published online: 5 June 2014

(C) Operations Research Society of China, Periodicals Agency of Shanghai University, and SpringerVerlag Berlin Heidelberg 2014

\begin{abstract}
Given a distribution of pebbles on the vertices of a connected graph $G$, a pebbling move on $G$ consists of taking two pebbles off one vertex and placing one on an adjacent vertex. The $t$-pebbling number $f_{t}(G)$ of a simple connected graph $G$ is the smallest positive integer such that for every distribution of $f_{t}(G)$ pebbles on the vertices of $G$, we can move $t$ pebbles to any target vertex by a sequence of pebbling moves. Graham conjectured that for any connected graphs $G$ and $H$, $f_{1}(G \times H) \leqslant f_{1}(G) f_{1}(H)$. Herscovici further conjectured that $f_{s t}(G \times H) \leqslant$ $f_{s}(G) f_{t}(H)$ for any positive integers $s$ and $t$. Wang et al. (Discret Math, 309: 3431$3435,2009)$ proved that Graham's conjecture holds when $G$ is a thorn graph of a complete graph and $H$ is a graph having the 2-pebbling property. In this paper, we further show that Herscovici's conjecture is true when $G$ is a thorn graph of a complete graph and $H$ is a graph having the $2 t$-pebbling property.
\end{abstract}

Keywords Thorn graph $\cdot t$-Pebbling number · Graham's conjecture · Herscovici's conjecture

\section{Introduction}

Pebbling in graphs was first studied by Chung [1]. Let $G$ be a simple graph with vertex set $V(G)$ and edge set $E(G)$. Considering a pebble distribution $p$ on the vertices of $G, p(v)$ denotes the number of pebbles on $v$ for any vertex $v$ of $G$, $p(H)=\sum_{v \in V(H)} p(v)$, where $H$ is the subgraph of $G$. A pebbling move consists of

This research was supported by National Natural Science Foundation of China (No. 11161016).

D.-L. Hao $\cdot$ Z.-T. Gao $(\bowtie) \cdot$ J.-H. Yin

Department of Mathematics, College of Information Science and Technology, Hainan University, Haikou 570228, China

e-mail: gaozetu@hainu.edu.cn 
removing two pebbles from one vertex and then placing one pebble at an adjacent vertex. The pebbling number of a vertex $v$ in a graph $G$ is the smallest positive integer $f(G, v)$ such that every distribution of $f(G, v)$ pebbles on the vertices of $G$, we can move one pebble to $v$ by a sequence of pebbling moves. The pebbling number $f(G)$ of a simple connected graph $G$ is the maximum of $f(G, v)$ over all vertices $v$. Similarly, the t-pebbling number of a vertex $v$ in a graph $G$ is the smallest positive integer $f_{t}(G, v)$ such that every distribution of $f_{t}(G, v)$ pebbles on the vertices of $G$, we can move $t$ pebbles to $v$ by a sequence of pebbling moves. The $t$ pebbling number $f_{t}(G)$ of a simple connected graph $G$ is the maximum of $f_{t}(G, v)$ over all vertices $v$. Evidently, $f_{1}(G)=f(G)$.

We say a graph $G$ has the 2-pebbling property if two pebbles can be moved to any specified vertex when the total starting number of pebbles is $2 f(G)-q+1$, where $q$ is the number of vertices with at least one pebble. Similarly, a graph $G$ has the $2 t$-pebbling property if $2 t$ pebbles can be moved to any specified vertex when the total starting number of pebbles is $2 f_{t}(G)-q+1$, where $q$ is the number of vertices with at least one pebble. Lourdusamy et al. [8,9] have proved that the star graph, the $n$-cube, the complete graph, the complete multi-partite graph, the fan graph, the wheel graph, and the even cycle have the $2 t$-pebbling property.

Conjecture 1.1 (Graham [1]) For any connected graphs $G$ and $H, f(G \times H) \leqslant$ $f(G) f(H)$.

Many articles (see, e.g., [1-3, 5, 10-12]) have given evidences supporting Conjecture 1.1. Recently, Herscovici further extended Conjecture 1.1 as follows:

Conjecture 1.2 (Herscovici [6]) For any connected graphs $G$ and $H, f_{s t}(G \times H)$ $\leqslant f_{s}(G) f_{t}(H)$.

Gao and Yin [4] proved that if $G$ is a tree, or a complete graph, or an even cycle and $H$ has the $2 t$-pebbling property, then Conjecture 1.2 holds.

Kirlangic [7] defined a thorn graph of the graph $G$ as follows: Let $G$ be a graph with $V(G)=\left\{v_{1}, v_{2}, \cdots, v_{n}\right\}$ and $p_{1}, \cdots, p_{n}$ be positive integers with $p_{i} \geqslant 1$ for each $i$. The thorn graph of $G$, with parameters $p_{1}, p_{2}, \cdots, p_{n}$, is obtained by attaching $p_{i}$ new vertices of degree 1 to vertex $v_{i}$ of $G$ for $i \in\{1,2, \cdots, n\}$. The thorn graph of $G$ is denoted by $G^{*}$ or $G^{*}\left(p_{1}, p_{2}, \cdots, p_{n}\right)$. When $G=K_{n}$, Wang et al. [12] proved the following three theorems:

Theorem 1.1 ([12]) If $n \geqslant 2$ and $p_{i} \geqslant 2$ for each $i$, then $f\left(K_{n}^{*}\right)=\sum_{i=1}^{n} p_{i}+2 n+2$.

Theorem 1.2 ([12]) If $p_{i} \geqslant 2$ for each $i$, then $K_{n}^{*}$ has the 2-pebbling property.

Theorem 1.3 ([12]) If $G$ has the 2-pebbling property and $p_{i} \geqslant 2$ for each $i$, then

$$
f\left(K_{n}^{*} \times G\right) \leqslant f\left(K_{n}^{*}\right) f(G) .
$$

The purpose of this paper is to extend the above theorems as follows:

Theorem 1.4 If $n \geqslant 2$ and $p_{i} \geqslant 1$ for each $i$, then $f_{t}\left(K_{n}^{*}\right)=\sum_{i=1}^{n} p_{i}+2 n+8 t-6$. 
Theorem 1.5 If $p_{i} \geqslant 1$ for each $i$, then $K_{n}^{*}$ has the $2 t$-pebbling property.

Theorem 1.6 If $G$ has the $2 t$-pebbling property and $p_{i} \geqslant 1$ for each $i$, then

$$
f_{s t}\left(K_{n}^{*} \times G\right) \leqslant f_{s}\left(K_{n}^{*}\right) f_{t}(G) .
$$

Corollary $\quad 1.1 \quad f_{s t}\left(K_{n}^{*} \times K_{m}^{*}\right) \leqslant f_{s}\left(K_{n}^{*}\right) f_{t}\left(K_{m}^{*}\right)=\left(\sum_{i=1}^{n} p_{i}+2 n+8 s-6\right)\left(\sum_{i=1}^{m} p_{i}+\right.$ $2 m+8 t-6)$

\section{Lemmas}

In this section, we present some lemmas that will be used in later proof. We always assume that $u_{i, 1}, u_{i, 2}, \cdots u_{i, p_{i}}$ are the $p_{i}$ vertices of degree 1 attached to $v_{i}$ for each $i$.

Given a configuration of pebbles placed on $G$, a transmitting subgraph of $G$ is defined to be a path $x_{1}, \cdots, x_{n}$ such that there are at least two pebbles on $x_{1}$ and at least one pebble on each of the other vertices in the path except $x_{n}$, so that one pebble can be moved to $x_{n}$. A tree $T$ can be viewed as a directed tree $\boldsymbol{T}_{v}$ with edges directed toward a root vertex $v$. A path-partition $P=\left\{\boldsymbol{P}_{1}, \cdots, \boldsymbol{P}_{n}\right\}$ of $\boldsymbol{T}_{v}$ is a set of edgedisjoint directed paths, the union of which is $\boldsymbol{T}_{v}$. We will assume that $\left|E\left(\boldsymbol{P}_{i}\right)\right| \geqslant$ $\left|E\left(\boldsymbol{P}_{j}\right)\right|$ whenever $i \leqslant j$. A path-partition $\left\{\boldsymbol{P}_{1}, \cdots, \boldsymbol{P}_{n}\right\}$ of $\boldsymbol{T}_{v}$ is said to majorize another $\left\{\boldsymbol{P}_{\boldsymbol{1}}^{\prime}, \cdots, \boldsymbol{P}_{\boldsymbol{n}}^{\prime}\right\}$ if the non-increasing sequence of its path size majorizes that of the other. That is, if $a_{i}=\left|E\left(\boldsymbol{P}_{i}\right)\right|$ and $b_{j}=\left|E\left(\boldsymbol{P}_{j}^{\prime}\right)\right|$, then $\left(a_{1}, \cdots, a_{n}\right)>\left(b_{1}, \cdots, b_{n}\right)$ if and only if $a_{i}>b_{i}$ where $i=\min \left\{j: a_{j} \neq b_{j}\right\}$. A path-partition of $\boldsymbol{T}_{v}$ is said to be maximum if it majorizes all other path-partitions of $\boldsymbol{T}_{v}$.

Lemma 2.1 ([1, 10]) If $a_{1}, \cdots, a_{m}$ is the sequence of the path sizes in a maximum path-partition of $\boldsymbol{T}_{v}$, then $f(T, v)=\sum_{i=1}^{m} 2^{a_{i}}-m+1$, and $f_{t}(T, v)=t 2^{a_{1}}+\sum_{i=2}^{m} 2^{a_{i}}-$ $m+1$.

Lemma 2.2 ([9]) $f_{t}\left(K_{n}\right)=2 t+n-2$, where $n \geqslant 2$.

Lemma 2.3 ([5]) Let $x_{i} x_{j} \in E(G)$. Suppose that in $G \times H$, we have $p_{i}$ pebbles occupying vertex $\left\{x_{i}\right\} \times H$, and $r_{i}$ of these vertices have an odd number of pebbles. If $r_{i} \leqslant k \leqslant p_{i}$ and if $k$ and $p_{i}$ have the same parity, then $k$ pebbles can be retained on $\left\{x_{i}\right\} \times H$, while moving $\frac{p_{i}-k}{2}$ pebbles onto $\left\{x_{j}\right\} \times H$. If $k$ and $p_{i}$ have the opposite parity, we must leave $k+1$ pebbles on $\left\{x_{i}\right\} \times H$, while moving $\frac{p_{i}-k-1}{2}$ pebbles onto $\left\{x_{j}\right\} \times H$. In particular, we can always move at least $\frac{p_{i}-r_{i}}{2}$ pebbles onto $\left\{x_{j}\right\} \times H$, since $p_{i}$ and $r_{i}$ have the same parity.

Lemma 2.4 ([4]) Let $T$ be a tree and $H$ have the 2t-pebbling property. For all positive integers $s$ and $t$, all vertices $x \in V(G)$ and $y \in V(H)$, we have

$$
f_{s t}(T \times H,(x, y)) \leqslant f_{s}(T, x) f_{t}(H) .
$$


Lemma 2.5 ([4]) Let $K_{n}$ be a complete graph and $G$ have the 2t-pebbling property. For all positive integers $s$ and $t$, we have

$$
f_{s t}\left(K_{n} \times G\right) \leqslant f_{s}\left(K_{n}\right) f_{t}(G) \text {. }
$$

\section{Proofs of Theorem 1.4-1.6}

Proof of Theorem 1.4 Considering the following distribution of $\sum_{i=1}^{n} p_{i}+2 n+$ $8 t-7$ pebbles on $K_{n}^{*}$ as follows: $p\left(u_{1,1}\right)=8 t-1, p\left(u_{1, j}\right)=1$ for all $j \in\left\{2, \cdots, p_{1}\right\}, p\left(u_{i, 1}\right)=3$ for all $i \in\{2, \cdots, n-1\}, p\left(u_{n, 1}\right)=0, p\left(u_{i, j}\right)=1$ for all $i \in\{2, \cdots, n-1\}$ and all $j \in\left\{2, \cdots, p_{i}\right\}, p\left(u_{n, j}\right)=1$ for all $j \in\left\{2, \cdots, p_{i}\right\}$, and $p\left(v_{i}\right)=0$ for all $i \in\{1, \cdots, n\}$. Then, $t$ pebbles can not be moved to $u_{n, 1}$. Thus, $f_{t}\left(K_{n}^{*}\right) \geqslant \sum_{i=1}^{n} p_{i}+2 n+8 t-6$. Now considering any distribution of $\sum_{i=1}^{n} p_{i}+$ $2 n+8 t-6$ pebbles on $K_{n}^{*}$. Let $s$ be the number of occupied vertices of $\left\{u_{1,1}, \cdots, u_{1, p_{1}}, u_{2,1} \cdots, u_{2, p_{2}}, u_{n, 1}, \cdots, u_{n, p_{n}}\right\}$. Clearly, $s \leqslant \sum_{i=1}^{n} p_{i}$. We will proceed by induction on $t$.

First, we assume $t=1$. Without loss of generality, we consider the following cases:

Case 1.1 The target vertex is $v_{1}$ and $p\left(v_{1}\right)=0$.

Let $p\left(K_{n}\right)=m$, we can assume that $0 \leqslant m \leqslant n-1$ by Lemma 2.2. By pebbling moves, we can move $\frac{1}{2}\left(\sum_{i=1}^{n} p_{i}+2 n+2-m-s\right)$ pebbles to $v_{i}$ from $u_{i, j}$ for $i \in\{1, \cdots, n\}, j \in\left\{1, \cdots, p_{i}\right\}$, and we have

$$
m+\frac{1}{2}\left(\sum_{i=1}^{n} p_{i}+2 n+2-m-s\right) \geqslant \frac{1}{2}\left(\sum_{i=1}^{n} p_{i}+2 n+2+m-s\right)>n
$$

pebbles on $K_{n}$. Hence, we can move one pebble to $v_{1}$ by Lemma 2.2.

Case 1.2 The target vertex is $u_{1,1}$ and $p\left(u_{1,1}\right)=0$.

Clearly, $s \leqslant \sum_{i=1}^{n} p_{i}-1$. By Lemma 2.2 , we can assume that $p\left(K_{n}\right)=m$, $0 \leqslant m \leqslant n+2-1$. By pebbling moves, we can move $\frac{1}{2}\left(\sum_{i=1}^{n} p_{i}+2 n+2-m-\right.$ $s)$ pebbles to $K_{n}$ from $u_{i, j}$ for each $i$ and each $j$ except $i=j=1$. Thus, at least

$$
m+\frac{1}{2}\left(\sum_{i=1}^{n} p_{i}+2 n+2-m-s\right) \geqslant \frac{1}{2}\left(\sum_{i=1}^{n} p_{i}+2 n+2+m-s\right) \geqslant n+\frac{3}{2}
$$

pebbles can be moved to $K_{n}$, this implies that $n+2$ pebbles can be moved to $K_{n}$, hence, we can move two pebbles to $v_{1}$ by Lemma 2.2. Furthermore, we can move one pebble to $u_{1,1}$.

Now, assume that Theorem 1.4 is true for $t \leqslant k(k \geqslant 1)$. We will prove that Theorem 1.4 is true for $t=k+1$. Without loss of generality, we consider the following cases:

Case 2.1 The target vertex is $v_{1}$ and $p\left(v_{1}\right)=\ell$ for $0 \leqslant l \leqslant k$.

If $1 \leqslant \ell \leqslant k$, then we can move $k+1-\ell$ additional pebbles to $v_{1}$ by the induction hypothesis, since $p\left(K_{n}^{*}-v_{1}\right)=\sum_{i=1}^{n} p_{i}+2 n+8(k+1)-6-\ell \geqslant \sum_{i=1}^{n} p_{i}+2 n+$ $8(k+1-\ell)-6$. Hence, we can move $k+1$ pebbles to $v_{1}$. Assume that $\ell=0$. By 
Lemma 2.2, we can assume that $p\left(K_{n}\right)=m, 0 \leqslant m \leqslant 2(k+1)+n-3$. By pebbling moves, we can move $\frac{1}{2}\left(\sum_{i=1}^{n} p_{i}+2 n+8(k+1)-6-m-s\right)$ pebbles to $K_{n}$ from $u_{i, j}$ for $i \in\{1, \cdots, n\}, j \in\left\{1, \cdots, p_{i}\right\}$. Thus, at least

$$
\begin{aligned}
m+\frac{1}{2}\left(\sum_{i=1}^{n} p_{i}+2 n+8(k+1)-6-m-s\right) & \geqslant \frac{1}{2}\left(\sum_{i=1}^{n} p_{i}+2 n+8(k+1)-6+m-s\right) \\
& \geqslant n+4(k+1)-3 \\
& >n+2(k+1)-2
\end{aligned}
$$

pebbles can be moved to $K_{n}$. Hence, we can move $k+1$ pebbles to $v_{1}$ by Lemma 2.2 .

Case 2.2 The target vertex is $u_{1,1}$ and $p\left(u_{1,1}\right)=\ell$.

If $1 \leqslant \ell \leqslant k$, then we can move additional $k+1-\ell$ pebbles to $u_{1,1}$ by the induction hypothesis, since $p\left(K_{n}^{*}-u_{1,1}\right)=\sum_{i=1}^{n} p_{i}+2 n+8(k+1)-6-\ell \geqslant \sum_{i=1}^{n} p_{i}+$ $2 n+8(k+1-\ell)-6$. Hence, we can move $k+1$ pebbles to $v_{1}$. Now suppose that $p\left(u_{1,1}\right)=0$. Clearly, $s \leqslant \sum_{i=1}^{n} p_{i}-1$. Let $p\left(K_{n}\right)=m$, we can assume that $0 \leqslant m \leqslant$ $4(k+1)+n-3$ by Lemma 2.2. By pebbling moves, we can move $\frac{1}{2}\left(\sum_{i=1}^{n} p_{i}+2 n+\right.$ $8(k+1)-6-m-s)$ pebbles to $K_{n}$ from $u_{i, j}$ for each $i$ and $j$ except $i=j=1$. Thus,

$$
\begin{aligned}
m+\frac{1}{2}\left(\sum_{i=1}^{n} p_{i}+2 n+8(k+1)-6-m-s\right) & \geqslant \frac{1}{2}\left(\sum_{i=1}^{n} p_{i}+2 n+8(k+1)-6+m-s\right) \\
& \geqslant 4(k+1)+n-\frac{5}{2} .
\end{aligned}
$$

This implies that $4(k+1)+n-2$ pebbles can be moved to $K_{n}$, and hence we can move $2(k+1)$ pebbles to $v_{1}$ by Lemma 2.2. Further, we can move $k+1$ pebbles to $u_{1,1}$.

Proof of Theorem 1.5 Clearly, $K_{n}^{*}$ is a star graph when $n=1$, and the result is true. Now, suppose that $n \geqslant 2$. Given a distribution of pebbles on $K_{n}^{*}$, let $p$ be the number of pebbles on $K_{n}^{*}, q$ be the number of vertices with at least one pebble, and $p=2\left(\sum_{i=1}^{n} p_{i}+2 n+8 t-6\right)-q+1$. Let $s$ be the number of occupied vertices of $\left\{u_{1,1}, \cdots, u_{1, p_{1}}, u_{2,1}, \cdots, u_{2, p_{2}}, u_{n, 1}, \cdots, u_{n, p_{n}}\right\}$, and $s^{\prime}$ be the number of occupied vertices of $\left\{v_{1}, v_{2}, \cdots, v_{n}\right\}$. Clearly, $s \leqslant \sum_{i=1}^{n} p_{i}, s^{\prime} \leqslant p\left(K_{n}\right), s+s^{\prime}=q$, and $q \leqslant \sum_{i=1}^{n} p_{i}+n$. Without loss of generality, we can assume that the target vertices are $v_{1}$ and $u_{1,1}$.

Case 1 The target vertex is $v_{1}$ and $p\left(v_{1}\right)=\ell$ for $0 \leqslant \ell \leqslant 2 t-1$.

If $\ell \geqslant 1$, then $p\left(K_{n}^{*}-v_{1}\right)=2\left(\sum_{i=1}^{n} p_{i}+2 n+8 t-6\right)-q+1-\ell \geqslant \sum_{i=1}^{n} p_{i}+$ $2 n+8(2 t-\ell)-6$. Thus, we can move $2 t-\ell$ additional pebbles to $v_{1}$ by Theorem 1.4. Hence, we can move $2 t$ pebbles to $v_{1}$. Assume that $p\left(v_{1}\right)=0$. Note that $q \leqslant \sum_{i=1}^{n} p_{i}+n-1$. Let $p\left(K_{n}\right)=m$, we can assume that $0 \leqslant m \leqslant 4 t+n-3$ by Lemma 2.2. By pebbling moves, we can move $\frac{1}{2}\left(2\left(\sum_{i=1}^{n} p_{i}+2 n+8 t-6\right)-q+\right.$ $1-m-s$ ) pebbles to $K_{n}$ from $u_{i, j}$ for each $i$ and $j$. Thus, at least 


$$
\begin{aligned}
& m+\frac{1}{2}\left(2\left(\sum_{i=1}^{n} p_{i}+2 n+8 t-6\right)-q+1-m-s\right) \geqslant \frac{1}{2}\left(2\left(\sum_{i=1}^{n} p_{i}+2 n+8 t-6\right)\right. \\
& -q+1+m-s) \geqslant 4 t+n-2
\end{aligned}
$$

pebbles on $K_{n}$. Hence, we can move $2 t$ pebbles to $v_{1}$ by Lemma 2.2 .

Case 2 The target vertex is $u_{1,1}$ and $p\left(u_{1,1}\right)=\ell$ for $0 \leqslant \ell \leqslant 2 t-1$.

If $\ell \geqslant 1$, then $p\left(K_{n}^{*}-u_{1,1}\right)=2\left(\sum_{i=1}^{n} p_{i}+2 n+8 t-6\right)-q+1-\ell \geqslant \sum_{i=1}^{n} p_{i}+$ $2 n+8(2 t-\ell)-6$. Thus, we can move $2 t-\ell$ additional pebbles to $u_{1,1}$ by Theorem 1.4. Assume that $p\left(u_{1,1}\right)=0$. Then, $s \leqslant \sum_{i=1}^{n} p_{i}-1$. Let $p\left(K_{n}\right)=m$, we can assume that $0 \leqslant m \leqslant 8 t+n-3$ by Lemma 2.2. By pebbling moves, then at least

$$
\begin{aligned}
m+\sum_{i=1}^{n} p_{i}+2 n+8 t-6+\frac{1-q-m-s}{2} \geqslant & \sum_{i=1}^{n} p_{i}+2 n+8 t-6 \\
& +\frac{1+m-\left(s+s^{\prime}\right)-s}{2} \\
& \geqslant 8 t+n-\frac{5}{2}
\end{aligned}
$$

pebbles can be moved to $K_{n}$, and this implies that $n+8 t-2$ pebbles can be moved to $K_{n}$. Hence, we can move $4 t$ pebbles to $v_{1}$ by Lemma 2.2. Therefore, we can move $2 t$ pebbles to $u_{1,1}$.

Proof of of Theorem 1.6 Clearly, $K_{n}^{*}$ is a tree when $n=1$, and so the result is true by Lemma 2.4. Now, suppose that $n \geqslant 2$. We consider any distribution of $\left(\sum_{i=1}^{n} p_{i}+2 n+8 s-6\right) f_{t}(G)$ pebbles on the vertices of $K_{n}^{*} \times G$. Let $G_{i j}=\left\{u_{i j}\right\} \times$ $G, m_{i j}$ be the number of pebbles on $G_{i j}$, and $r_{i j}$ be the number of vertices with an odd number of pebbles in $G_{i j}$ for each $i$ and each $j$.

Case 1 The target vertex is $\left(v_{i}, y\right)$ for some $y \in V(G)$.

Without loss of generality, assume that the target vertex is $\left(v_{1}, y\right)$. Clearly, $K_{n}^{*}-\left\{u_{1,1}, \cdots, u_{1, p_{1}}, u_{2,1} \cdots, u_{2, p_{2}}, u_{n, 1}, \cdots, u_{n, p_{n}}\right\}=K_{n}$. By $r_{i j} \leqslant|V(G)| \leqslant f(G) \leqslant$ $f_{t}(G), \sum_{i=1}^{n} \sum_{j=1}^{p_{i}} m_{i j} \leqslant\left(\sum_{i=1}^{n} p_{i}+2 n+8 s-6\right) f_{t}(G)$, we get

$$
\begin{aligned}
\sum_{i=1}^{n} \sum_{j=1}^{p_{i}}\left(m_{i j}+r_{i j}\right) & =\sum_{i=1}^{n} \sum_{j=1}^{p_{i}} m_{i j}+\sum_{i=1}^{n} \sum_{j=1}^{p_{i}} r_{i j} \\
& \leqslant\left(\sum_{i=1}^{n} p_{i}+2 n+8 s-6\right) f_{t}(G)+\left(\sum_{i=1}^{n} p_{i}\right) f_{t}(G) \\
& =2\left(\sum_{i=1}^{n} p_{i}+n+4 s-3\right) f_{t}(G) .
\end{aligned}
$$

By Lemma 2.3, we can move at least $\sum_{i=1}^{n} \sum_{j=1}^{p_{i}}\left(\frac{m_{i j}-r_{i j}}{2}\right)$ pebbles to $K_{n} \times G$ from all the vertices in $G_{11}, \cdots, G_{1 p_{1}}, \cdots, G_{n 1}, \cdots, G_{n p_{n}}$. So, we have at least 


$$
\begin{aligned}
& \left(\sum_{i=1}^{n} p_{i}+2 n+8 s-6\right) f_{t}(G)-\sum_{i=1}^{n} \sum_{j=1}^{p_{i}} m_{i j}+\sum_{i=1}^{n} \sum_{j=1}^{p_{i}}\left(\frac{m_{i j}-r_{i j}}{2}\right) \\
& =\left(\sum_{i=1}^{n} p_{i}+2 n+8 s-6\right) f_{t}(G)-\sum_{i=1}^{n} \sum_{j=1}^{p_{i}}\left(\frac{m_{i j}+r_{i j}}{2}\right) \\
& \geqslant\left(\sum_{i=1}^{n} p_{i}+2 n+8 s-6\right) f_{t}(G)-\left(\sum_{i=1}^{n} p_{i}+n+4 s-3\right) f_{t}(G) \\
& =(4 s+n-3) f_{t}(G) \\
& \geqslant(2 s+n-2) f_{t}(G)
\end{aligned}
$$

pebbles on $K_{n} \times G$. Therefore, we can move st pebbles to $\left(v_{1}, y\right)$ by Lemma 2.5.

Case 2 The target vertex is $\left(u_{i, j}, y\right)$ for some $y \in V(G)$.

Without loss of generality, assume that the target vertex is $\left(u_{1,1}, y\right)$. Let $T$ be a tree obtained from $K_{n}^{*}$ by deleting all edges between $v_{i}$ and $v_{j}$ for $i, j \in\{2, \cdots, n\}$. By Lemma 2.1, we have $f_{s}\left(T, u_{1,1}\right)=\sum_{i=1}^{n} p_{i}+2 n+8 s-6$. Note that $T \times G$ is a spanning subgraph of $K_{n}^{*} \times G$ and $f_{s t}\left(K_{n}^{*} \times G,\left(u_{1,1}, y\right)\right) \leqslant f_{s t}\left(T \times G,\left(u_{1,1}, y\right)\right)$. Hence, by Lemma 2.4, we have

$$
\begin{aligned}
f_{s t}\left(K_{n}^{*} \times G,\left(u_{1,1}, y\right)\right) & \leqslant f_{s t}\left(T \times G,\left(u_{1,1}, y\right)\right) \leqslant f_{s}\left(T, u_{1,1}\right) f_{t}(G) \\
& =\left(\sum_{i=1}^{n} p_{i}+2 n+8 s-6\right) f_{t}(G) .
\end{aligned}
$$

Therefore, we can move st pebbles to $\left(u_{1,1}, y\right)$.

Acknowledgments The authors are grateful to the referees for their helpful suggestions and their careful reading of this paper.

\section{References}

[1] Chung, F.R.K.: Pebbling in hypercubes. SIAM J. Discret. Math. 2, 461-472 (1998)

[2] Gao, Z.T., Yin, J.H., Li, W.Y.: Graham's pebbling conjecture on the product of generalized friendship graphs. Appl. Math. J. Chin. Univ. Ser. A 23, 487-491 (2008)

[3] Gao, Z.T., Yin, J.H.: Pebbling numbers of some bipartite graphs. Appl. Math. J. Chin. Univ. Ser. A 45, 365-371 (2010)

[4] Gao, Z.T., Yin, J.H.: On the $t$-pebbling number and the $2 t$-pebbling property of graphs. Discret. Appl. Math. 161, 999-1005 (2013)

[5] Herscovici, D.S.: Graham's pebbling conjecture on products of cycles. J. Graph Theory 42, 141-154 (2003)

[6] Herscovici, D.S.: Graham's pebbling conjecture on products of many cycles. Discret. Math. 308, 6501-6512 (2008)

[7] Kirlangic, A.: The scattering number of thorn graph. Int. J. Comput. Math. 82, 299-311 (2004)

[8] Lourdusamy, A.: $t$-pebbling the product of graphs. Acta Cienc. Indica 32(1), 171-176 (2006)

[9] Lourdusamy, A., Jeyaseelan, S.S., Tharani, A.P.: $t$-pebbling the product of fan graphs and the product of wheel graphs. Int. Math. Forum 32, 1573-1585 (2009)

[10] Moews, D.: Pebbling graphs. J. Comb. Theory Ser. B 55, 244-252 (1992)

[11] Wang, S.S.: Pebbling and Graham's conjecture. Discret. Math. 226, 431-438 (2001)

[12] Wang, Z.P., Zou, Y.T., Liu, H.Y., Wang, Z.G.: Graham's pebbling conjecture on product of thorn graphs of complete graphs. Discret. Math. 309, 3431-3435 (2009) 